\title{
Música más lengua de signos: una revisión de la literatura
}

\author{
Se Music in sign language: a literature review
}

\section{Música mais linguagem de sinais: uma revisão da literatura}

\author{
iD Nadal-García, Icíar ${ }^{1}$ \\ Universidad de Zaragoza, Zaragoza, España \\ iciarnad@unizar.es \\ iD López-Casanova, M. Belén² \\ Universidad de Zaragoza, Zaragoza, España \\ belocasa@unizar.es \\ Juan-Morera, Borja³ \\ Universidad de Zaragoza, Zaragoza, España \\ bjuan@unizar.es
}

Resumen: La Lengua de Signos incorporada a la música es un nuevo arte inclusivo que cada vez va ganando más presencia en la sociedad, debido a su difusión en las redes sociales y a diferentes iniciativas artísticas. Se concibe la música como una experiencia multisensorial en la que los estímulos visuales, táctiles y el movimiento expresivo enriquecen la experiencia auditiva. El objetivo de este estudio es conocer la incidencia de

\footnotetext{
1 Icíar Nadal-García - Doctora en Filosofía y Letras por la Universidad de Zaragoza. Titulada Superior en Pedagogía Musical, Dirección de Coros y Dirección de Orquesta y Solfeo y Teoría de la Música por el Conservatorio Superior de Zaragoza. Profesora Titular del Departamento de Expresión Musical, Plástica y Corporal de la Universidad de Zaragoza. Miembro del grupo de Investigación H05_17R: HERAF: Hermenéutica y Antropología Fenomenológica. Fundadora y coordinadora del coro inclusivo Cantatutti de la Universidad de Zaragoza, que obtuvo en 2019 el Premio Impulso Solidario de la Ibercaja y en 2019 el Premio Ebrópolis a las Buenas Prácticas Ciudadanas. Co-directora de la Cátedra: Música e Inclusión para el Cambio Social.

2 M. Belén López-Casanova - Doctora por la Universidad de Zaragoza. Título Superior de solfeo y teoría de la música, por el Conservatorio de Música de Zaragoza. Profesora Titular del Departamento de Expresión Musical, Plástica y Corporal de la Universidad de Zaragoza. Directora del primer postgrado en musicoterapia de la Universidad de Zaragoza. Fundadora y coordinadora del coro inclusivo Cantatutti de la Universidad de Zaragoza, que obtuvo en 2019 el Premio Impulso Solidario de la Ibercaja y el Premio Ebrópolis a las Buenas Prácticas Ciudadanas. Actualmente desarrolla su actividad profesional e investigadora en la Universidad de Zaragoza como miembro colaborador del grupo de investigación ETNOEDU.

3 Borja Juan-Morera - Título Superior de piano por el Conservatorio Superior de Música de Aragón. Mejor Trabajo Final del Máster en Investigación Predoctoral, Universidad de Zaragoza. Actualmente desarrolla su doctorado en Música e Inclusión para el Cambio Social. Director y fundador del coro inclusivo Cantatutti de la Universidad de Zaragoza, ganador del $2^{\circ}$ premio "Impulso solidario" de la Fundación Ibercaja. Ganador del premio "Ebrópolis" a las buenas prácticas ciudadanas en diciembre de 2019. Durante ocho años, director del Ensamble y Coro Ruta Quetzal BBVA, con actuaciones en diversos escenarios de Europa y Latinoamérica.
} 
la lengua de signos aplicada a la interpretación musical en sus diferentes modalidades, así como evidenciar sus aportaciones a la percepción musical de las personas sordas y oyentes a través de una revisión de la literatura académica existente. La búsqueda se realiza a partir de bases de datos especializadas, Web of Science (WoS), Scopus-Elsevier (Scopus), ProQuest, y el buscador digital Google Académico en español y en inglés. Se establecen unos criterios de inclusión y exclusión con el fin de obtener los documentos relacionados con el objetivo de estudio. En los resultados obtenidos se localizan escasez de estudios y la mayor parte de los documentos tratan de experiencias personales o analizan videos de YouTube. Se verifica que la amalgama de música más Lengua de Signos es una práctica inclusiva multimodal que hace que las experiencias perceptivas sean más significativas para las personas con o sin discapacidad auditiva. Al mismo tiempo, se evidencia que otros presuntos beneficios de esta práctica están todavía pendientes de estudio.

Palabras clave: música, lengua de signos, educación inclusiva, educación musical, percepción musical.

Abstract: Sign Language incorporated into music is a new kind of inclusive art that is getting more and more presence in society due to social networks and different artistic initiatives. Music is conceived as a multisensory experience in which visual and tactile stimuli and expressive movement enrich the listening experience. This work aims at knowing the incidence of sign language in musical interpretation, as well as verifying its possible contributions to the musical perception of people with or without hearing impairment through a review of the academic literature. The search was carried out using specialized databases, Web of Science (WoS), Scopus-Elsevier (Scopus), ProQuest, and the Google Scholar digital search engine in Spanish and English. Inclusion and exclusion criteria are established in order to exclusively obtain documents related to the study objective. In the results obtained, there is a scarcity of studies, most of the documents deal with personal experiences or analyze YouTube videos. Verifies that the amalgamation of music plus Sign Language is an inclusive multimodal practice that makes perceptual experiences more meaningful for people with or without hearing impairment. At the same 
time, it is evident that other presumed benefits of this practice are still pending study.

Keywords: music, sign language, inclusive education, musical education, musical perception.

Resumo: A Linguagem de Sinais incorporada à música é uma nova arte inclusiva que está ganhando cada vez mais presença na sociedade, devido à sua divulgação nas redes sociais e diversas iniciativas artísticas. A música é concebida como uma experiência multissensorial na qual estímulos visuais e táteis e movimentos expressivos enriquecem a experiência de escuta. O objetivo deste estudo é conhecer a incidência da língua de sinais aplicada à interpretação musical em suas diferentes modalidades, bem como demonstrar suas contribuições para a percepção musical de surdos e ouvintes, por meio de uma revisão da literatura acadêmica existente. A pesquisa é realizada a partir de bases de dados especializadas Web of Science (WoS), Scopus-Elsevier (Scopus), ProQuest e do motor de busca digital Google Academic em espanhol e inglês. Critérios de inclusão e exclusão são estabelecidos para a obtenção dos documentos relacionados ao objetivo do estudo.

Os resultados obtidos revelam uma escassez de estudos e a maioria dos documentos trata de experiências pessoais ou analisa vídeos do YouTube. Verifica-se que o amálgama música mais Língua de Sinais é uma prática multimodal inclusiva que torna as experiências perceptivas mais significativas para pessoas com ou sem deficiência auditiva. Ao mesmo tempo, é evidente que outros supostos benefícios dessa prática ainda estão pendentes de estudo.

Palavras-chave: música, língua de sinais, educação inclusiva, educação musical, percepção musical.

Submetido em: 8 de fevereiro de 2021

Aceito em: 5 de julho de 2021 


\section{Introducción}

La música es una experiencia universal para todos los seres humanos. Las personas sordas deben de tener las mismas oportunidades de educación y disfrute de la música que el resto de los ciudadanos y no pueden estar al margen de esta práctica. En la carta pública expuesta con motivo del XVIII Congreso Mundial de Personas Sordas (2019), se manifiesta que ha habido un "cambio de paradigma" de un modelo médico de discapacidad a un modelo de derechos humanos, en el que las personas sordas tienen las mismas oportunidades para participar en la sociedad que el resto. La música como arte inclusivo y universal debe estar preparada ante este reto. Tejada $(2004$, p.18) sugiere que "una forma más natural y coherente de educación musical en nuestra sociedad contemporánea debería tratar de resolver las discrepancias del mundo en que vivimos: armonizar las diferencias individuales".

Las descripciones que encontramos a lo largo de la historia del término "música" han enfatizado la estética auditiva, 'ya que los procesos que apoyan la interpretación musical se centran, sobre todo, en la audición (BEST, 2018). Es necesario que el significado del término "música" incluya el relato de la experiencia de los Sordos ${ }^{4}$, y vaya más allá de las experiencias auditivas (MALER, 2015). Existen diferentes técnicas y procedimientos a la hora de percibir la música. El sonido no solo se experimenta a través de los oídos, puede sentirse de manera táctil o experimentarlo visualmente, o incluso como una idea (KIM, 2015). El sonido es mucho más de lo que se escucha con el oído; se trata de una experiencia multisensorial. Las personas sordas se han implicado desde hace mucho tiempo con la música a través de estímulos táctiles, visuales y cinestésicos como alternativa a la audición normal (HOLMES, 2017).

La práctica de la Lengua de Signos (en adelante, LS) aplicada a la interpretación musical, es un fenómeno multimodal (MALER,

4 El término Sordo/a con mayúscula, según Serrat y Fernández-Viader (2013), se utiliza para describir a las personas que se identifican con una comunidad lingüística-cultural, mientras que el concepto de sordo con minúscula tiene un uso genérico sin connotaciones sociales. 
2013; CRIPPS, 2018; PEÑALBA, MORIYÓN Y LUQUE, 2018), que va más allá de lo sonoro y que permite hacer accesible la música a personas sordas, sin olvidar la importancia de su grado de déficit auditivo, antecedentes educativos, culturales, etc. "Los intérpretes de música en LS transmiten un mensaje, una intención y un sentimiento que llega de forma directa al espectador, se convierten en artistas. Se trata de una nueva forma de escuchar y hacer música donde no sólo participa el fenómeno sonoro" (JUAN, NADAL Y LÓPEZ, 2020, p.38).

Según constata la Canadian Cultural Society of the Deaf (2015), la música signada es un arte que surge de la comunidad Sorda, y puede incorporar características poéticas literarias de la LS añadiendo elementos de la música expresados como una forma artística visual y utilizando diferentes formas de interpretación para lograr su fin: danza, poesía y elementos musicales visuales. La comunidad Sorda es capaz de apreciar las experiencias de la música signada, pues siguiendo las aportaciones de los estudios realizados por diversos autores, esta música signada es real y significativa para las personas que conocen la LS (CRIPPS y LYONBLUM, 2017). En el año 2004 Curtis y Mercado analizaron cómo la música en LS iba ganando popularidad, primero en las personas sordas y luego en los oyentes. El poder evocador de la música se ve enormemente mejorado por la conmovedora belleza de la LS (Curtis, 2004).

Las personas sordas son grandes consumidoras de los medios digitales (SERRAT y FERNÁNDEZ-VIADER, 2013). Las redes sociales han sido una herramienta eficaz para ayudar a la comunidad Sorda a participar en las actividades de la sociedad en general. El hecho de poder difundir estos materiales a través de las redes sociales permite a los Sordos compartir su "voz", su propia lengua y crear un entorno inclusivo (MAULIDA, NAYLILHUSNA y ANTULA, 2019), pues desarrolla la concienciación por la discapacidad en las personas oyentes que encuentran una nueva forma de estrechar relaciones con la comunidad Sorda.

El propósito de este estudio es conocer la incidencia de la LS aplicada a la interpretación musical en sus diferentes modalidades, 
así como evidenciar sus aportaciones a la percepción musical de las personas sordas y oyentes, a través de una revisión de la literatura académica existente.

\section{Método}

El trabajo de revisión realizado es de tipo descriptivo, de esta forma se puede actualizar el estado del tema objetivo de estudio y conocer las aportaciones de las investigaciones realizadas.

Las búsquedas de los documentos se realizaron en español e inglés en tres de las principales bases de datos internacionales: Web of Science (Wos), Scopus-Elvesier (SCOPUS) y ProQuest, a través del buscador digital Google Académico y de forma manual. El proceso de búsqueda se desarrolló entre los meses de marzo de 2020 a junio de 2020 . Se seleccionaron las publicaciones que relacionan la música con la LS tanto si eran, o no, de investigaciones empíricas. En la indagación llevada a cabo se requirió que, en el resumen, en el título o en las palabras clave aparecieran alguno de los siguientes términos en español: "música signada", "canciones signadas", "música en lengua de signos", "canciones en lengua de signos", "música y cultura Sorda". Y en inglés: "signed music", "signed songs", "music and sign language", "songs and sign language", "Music and Deaf". Para la realización de las búsquedas se introdujo el operador booleano AND para así adquirir mayor número de referencias.

\section{Criterios de inclusión y exclusión}

Los criterios de inclusión y exclusión establecidos permitieron descartar documentos que pudieran contemplar las palabras empleadas para la búsqueda, pero sin embargo se alejaran del objetivo de estudio. 
Música más lengua de signos: una revisión de la literatura

Nadal-García, Icíar · López-Casanova, M. Belén ·Juan-Morera, Borja

\section{Tabla 1- Criterios de inclusión y exclusión}

\begin{tabular}{|l|l|l|}
\hline \multicolumn{1}{|c|}{ Criterio } & \multicolumn{1}{|c|}{ Inclusión } & \multicolumn{1}{c|}{ Exclusión } \\
\hline Periodo & Publicaciones entre 1970 y 2020 & $\begin{array}{l}\text { Publicaciones } \\
\text { anteriores a 1970 } \\
\text { Temática }\end{array}$ \\
$\begin{array}{l}\text { Tipo de docu- } \\
\text { mento }\end{array}$ & $\begin{array}{l}\text { Música vocal o instrumental y su re- } \\
\text { presentación en LS } \\
\text { Artículos, capítulo de libro, } \\
\text { Tesis de maestría o de máster de in- } \\
\text { vestigación, Actas de congresos y TFMs } \\
\text { de investigación }\end{array}$ & $\begin{array}{l}\text { Artículos de periódico, TFGs, TFMs } \\
\text { que no son de investigación }\end{array}$ \\
\hline Idioma & $\begin{array}{l}\text { Inglés y español } \\
\end{array}$ & $\begin{array}{l}\text { Cualquier idioma que no sea inglés } \\
\text { o español }\end{array}$ \\
\hline
\end{tabular}

Fuente: elaboración propia.

La fecha de inicio se estableció en el año 1970 debido a la escasa documentación que existe sobre la materia. La temática se acotó exclusivamente a la LS (de cualquier idioma) aplicada a la música vocal o instrumental. Se excluyeron los documentos que trataban sobre la música y la cultura Sorda o discapacidad auditiva, y en su contenido tan apenas abordaban el objetivo de estudio. En cuanto al tipo de documentos, se descartaron los que tenían características más divulgativas y menos científicas. Los idiomas seleccionados fueron el español y el inglés.

\section{Procedimiento de revisión}

Inicialmente, la búsqueda de documentos se llevó a cabo por tres investigadores de forma independiente, para así poder contrastar las búsquedas localizadas por cada uno de ellos. Estas primeras búsquedas se realizaron en Scopus, WoS, ProQuest y Google Académico. Se tuvo en cuenta que en el título, palabras clave o resumen aparecieran los términos acordados. Una vez seleccionados los documentos resultantes, se analizaron los resúmenes para constatar que la temática estaba relacionada con el objeto de estudio. En esta fase se obtuvieron 199 documentos, pero 183 
Música más lengua de signos: una revisión de la literatura

Nadal-García, Icíar · López-Casanova, M. Belén ·Juan-Morera, Borja

se excluyeron al no superar los criterios de inclusión. Finalmente pasaron la criba 16 documentos.

Tabla 2 - Clasificación de los resultados de búsqueda

\begin{tabular}{|c|c|c|c|}
\hline \multicolumn{2}{|c|}{$\begin{array}{l}\text { Bases de datos } \\
\text { Fuentes documentales }\end{array}$} & \multirow{2}{*}{$\begin{array}{l}\text { Incluidos } \\
4 \text { ( } 1 \text { coincide con WoS y Scopus) }\end{array}$} & \multirow{2}{*}{$\begin{array}{l}\text { Excluidos } \\
15 \\
\end{array}$} \\
\hline Conolo orodómira & Español - 19 & & \\
\hline Googie academico & Inglés - 157 & 11 ( 2 coinciden con ProQuest) & 146 \\
\hline \multirow{2}{*}{ Scopus } & Español - 1 & 1 (coincide con WoS y GA) & \\
\hline & Inglés - 8 & 1(coincide con WoS y ProQuest) & 7 \\
\hline \multirow{2}{*}{ Web of Science } & Español - 1 & 1(coincide con Scopus y GA) & \\
\hline & Inglés - 9 & 1 (1 coincide con Scopus y Proquest; 2 con GA) & 8 \\
\hline \multirow{2}{*}{ ProQuest } & Español - 0 & & \\
\hline & Inglés - 10 & 3 (1 coincide con Scopus y WoS; 2 con GA) & 7 \\
\hline \multirow{2}{*}{ Búsqueda manual } & Español - 3 & 3 & \\
\hline & Inglés - 11 & 11 & \\
\hline \multicolumn{2}{|c|}{ Total de documentos } & 30 & 183 \\
\hline
\end{tabular}

Fuente: elaboración propia.

Durante el periodo en el que se desarrolló la investigación se consideraron otros documentos que los investigadores obtuvieron de forma manual y que aunque no se encontraron en las bases de datos, a través de las palabras clave acordadas sí que cumplían los criterios de inclusión. Los documentos se identificaron en actas de congresos relacionados con la LS y a partir de la revisión de la bibliografía de los artículos encontrados. De esta forma se obtuvieron 3 documentos en español y 11 en inglés.

A continuación, se procedió a la lectura del documento completo para garantizar que los artículos estaban dentro de la temática elegida. Los artículos seleccionados, un total de 30, se analizaron de forma sistemática con el objetivo de determinar el tipo de documento, la metodología empleada, el tema abordado y las principales aportaciones sobre el tema de estudio. 
Música más lengua de signos: una revisión de la literatura

Nadal-García, Icíar · López-Casanova, M. Belén ·Juan-Morera, Borja

\section{Resultados}

\section{Cuadro 1 - Cuadro descriptivo de los documentos analizados}

\begin{tabular}{|l|l|l|l|l|}
\hline Autor/Año & Idioma & \multicolumn{1}{c|}{$\begin{array}{c}\text { Tipo de documento/ } \\
\text { Búsqueda }\end{array}$} & $\begin{array}{c}\text { Método Investi- } \\
\text { gación }\end{array}$ & \multicolumn{1}{|c|}{ Tema } \\
\hline $\begin{array}{l}\text { Sutherland, } \\
\text { E. }\end{array}$ & Inglés & $\begin{array}{l}\text {-Artículo de revista cien- } \\
\text { tífica especializada en LS } \\
(\mathbf{1 9 8 5 )}\end{array}$ & Cualitativo & $\begin{array}{l}\text { Música interpretada- } \\
\text {-traducida a LS }\end{array}$ \\
\hline
\end{tabular}

La investigación parte de entrevistas realizadas a intérpretes de música en LS y espectadores sordos que asisten a un festival de música donde las canciones se traducen a LS.

El movimiento rítmico del cuerpo "el baile" realizado por los intérpretes de LS representa la música de forma visual.

Los intérpretes de música en LS transmiten ritmo, movimiento y emoción (elementos, a menudo ausentes en la comunicación oral). El intérprete aporta una experiencia conceptual y emocional tridimensional que experimenta, parcialmente, el público oyente. Concibe la interpretación de música en LS como una nueva profesión y acerca la cultura Sorda a los oyentes.

\begin{tabular}{|l|l|l|l|l|}
\hline $\begin{array}{l}\text { Darrow, } \\
\text { A-A \& Loo- } \\
\text { mis, D. M. } \\
\text { (1999) }\end{array}$ & Inglés & $\begin{array}{l}\text {-Artículo de revista cien- } \\
\text { tífica especializada en } \\
\text { musicoterapia. }\end{array}$ & Cualitativo & Música signada \\
& & $\begin{array}{l}\text {-Scopus,Wos y ProQuest } \\
\text { Contrasta }\end{array}$ & & \\
\hline
\end{tabular}

Contrasta impresiones de sordos y oyentes tras revisar interpretaciones de música en LS. Hacer que el ritmo sea visual aumenta enormemente la percepción musical de los estudiantes sordos. El movimiento corporal de los gestos de la LS indica el ritmo de la música, demostrando la ventaja de la representación visual.

\begin{tabular}{|c|c|c|c|c|}
\hline $\begin{array}{l}\text { Curtis, S. } \\
\text { L. \& } \\
\text { Mercado, } \\
\text { C.S. } \\
\text { (2004) }\end{array}$ & Inglés & $\begin{array}{l}\text {-Artículo de revista cien- } \\
\text { tífica especializada en } \\
\text { musicoterapia } \\
\text {-Google académico }\end{array}$ & Cualitativo & Música signada \\
\hline
\end{tabular}

Realizan su investigación a través de un programa de artes escénicas en el que participan personas con y sin diversidad funcional. Todos los involucrados disfrutaron de la pasión compartida por las artes escénicas, constatando que la diversidad aporta diferentes habilidades y dones y la idea de que, tal vez, somos más parecidos que diferentes.

\begin{tabular}{|l|l|l|l|l|}
\hline $\begin{array}{l}\text { Bahan, B. } \\
\text { (2006) }\end{array}$ & Inglés & $\begin{array}{l}\text {-Capítulo de libro espe- } \\
\text { cializado en sordos } \\
\text {-Manual }\end{array}$ & $\begin{array}{l}\text { Descriptivo/ana- } \\
\text { lítico } \\
\text { observacional }\end{array}$ & $\begin{array}{l}\text { Canciones signadas } \\
\text { Percusión signada }\end{array}$ \\
\hline
\end{tabular}

Sugiere dos tipos de música signada: canciones traducidas y canciones de percusión. Signar canciones de percusión es una práctica cada vez más extendida a lo largo de los años y puede estar resurgiendo como un medio para afianzar el sentimiento de grupo. La tecnología audiovisual influye en las composiciones y en la aparición de nuevos artistas-creadores en este ámbito, y es un valioso recurso que reemplaza, en muchas ocasiones, el face to face, que ha sido una práctica indispensable en la comunidad Sorda durante años. 
Música más lengua de signos: una revisión de la literatura

Nadal-García, Icíar · López-Casanova, M. Belén ·Juan-Morera, Borja

\begin{tabular}{|l|l|l|l|l|}
\hline $\begin{array}{l}\text { Maler, A. } \\
\text { (2013) }\end{array}$ & Inglés & $\begin{array}{l}\text {-Artículo de revista cien- } \\
\text { tífica especializada en } \\
\text { musicoterapia } \\
\text {-Google académico y } \\
\text { ProQuest }\end{array}$ & $\begin{array}{l}\text { Exploratoria/ } \\
\text { analítica }\end{array}$ & Música signada \\
\hline
\end{tabular}

Realiza una revisión teórica y un posterior análisis de videos de canciones signadas. Examina la interacción de la LS, las palabras cantadas y la música como elementos que dan forma a la comprensión de una canción. Constata como, al usar el signado, el artista es capaz de representar aspectos como los parámetros sonoros o los gestos no lingüísticos, enriqueciendo así la comprensión musical de sordos y oyentes.

\begin{tabular}{|l|l|l|l|l|}
\hline $\begin{array}{l}\text { Canadian } \\
\text { Cultural } \\
\text { Society of } \\
\text { the Deaf }\end{array}$ & Inglés & $\begin{array}{l}\text {-Volumen } \mathrm{n}^{\circ} 2 \text { de la } \\
\text { serie Arts Deaf } \\
\text { (2015) }\end{array}$ & $\begin{array}{l}\text { Descriptivo/ana- } \\
\text { lítico }\end{array}$ & Música signada \\
\hline
\end{tabular}

Describen las características de la música signada en la comunidad Sorda y narran, desde una perspectiva histórica, la evolución de la música signada en EE. UU. y en Canadá. Estudian tres tipos de música signada: 1. Música signada sin componentes auditivos (o que pueden añadirse después); 2. música signada inspirada en un componente auditivo-visual; 3. Música signada que incorpora la cinematografía como una característica artística de la actuación.

\begin{tabular}{|l|l|l|l|l|}
\hline $\begin{array}{l}\text { Maler, A. } \\
\text { (2015) }\end{array}$ & Inglés & $\begin{array}{l}\text {-Capítulo de libro de } \\
\text { serie dedicada a Música } \\
\text { y discapacidad } \\
\text {-Google Académico }\end{array}$ & $\begin{array}{l}\text { Exploratoria/ } \\
\text { analítica }\end{array}$ & Canciones signadas \\
\hline
\end{tabular}

Analiza las prácticas desarrolladas sobre canciones signadas en videos realizados por personas sordas y oyentes. Plantea la posibilidad de que la discapacidad auditiva puede ser una fuente de habilidad musical más que de discapacidad y que la audición incluso puede verse como una discapacidad en el contexto de la práctica musical sorda.

Los "cantantes sordos" en LS no solo traducen lo audible a lo visual; más bien crean música en sus representaciones visuales, es decir, crean espectáculos visuales. El concepto de "música" va más allá de escuchar experiencias. La expresión musical debe incluir las contribuciones de los sordos.

\begin{tabular}{|l|l|l|l|l|}
\hline $\begin{array}{l}\text { Andrade, F. } \\
(\mathbf{2 0 1 6 )}\end{array}$ & Español & -Tesis de maestría & $\begin{array}{l}\text { Descriptivo/Ana- } \\
\text { lítico }\end{array}$ & $\begin{array}{l}\text { Música sorda, su } \\
\text { representación }\end{array}$ \\
\hline
\end{tabular}

Apunta que puede surgir un nuevo género musical totalmente atípico, socio-estéticamente materializado si se musicalizan las intenciones comunicativas de los sordos y se explora cómo convertir el "canto sordo" en "canto audible".

\begin{tabular}{|l|l|l|l|l|}
\hline $\begin{array}{l}\text { Cripps, J. H. } \\
\text { Rosenblum, } \\
\text { E \& Small, } \\
\text { A. (2016) }\end{array}$ & Inglés & $\begin{array}{l}\text {-Capítulo de libro espe- } \\
\text { cializado en estudios } \\
\text { sobre sordos }\end{array}$ & $\begin{array}{l}\text { Descriptivo/Ana- } \\
\text { lítico }\end{array}$ & Música signada \\
\hline -Manual & & \\
\hline
\end{tabular}

Acuñan la definición de música signada, atribuyendo el término a la comunidad Sorda, pues defienden que sólo es comprensible en el contexto de las artes escénicas para sordos. La música signada está asociada a componentes viso-gestuales y multisensoriales. 
Música más lengua de signos: una revisión de la literatura

Nadal-García, Icíar · López-Casanova, M. Belén ·Juan-Morera, Borja

\begin{tabular}{|l|l|l|l|l|}
\hline $\begin{array}{l}\text { Egbert, L. D. } \\
\text { (2017) }\end{array}$ & Inglés & $\begin{array}{l}\text {-Artículo de revista } \\
\text { especializada en Lengua } \\
\text { de signos americana } \\
\text { (ASL) } \\
\text {-Manual }\end{array}$ & Descriptivo & Música signada \\
\hline
\end{tabular}

Expone cómo la cultura Sorda está dividida entre los que están a favor de la "música signada" y los que no. Presenta las teorías de los investigadores que en esos momentos trabajaban el tema y reclama que se ofrezca a los niños sordos la oportunidad de disfrutar y apreciar las artes visuales para su desarrollo personal.

\begin{tabular}{|l|l|l|l|l|}
\hline $\begin{array}{l}\text { Cripps, J. H., } \\
\text { \& Lyon- } \\
\text { blum, E. } \\
\text { (2017) }\end{array}$ & Inglés & $\begin{array}{l}\text {-Artículo de revista es- } \\
\text { pecializada en ASL } \\
\text {-Manual }\end{array}$ & $\begin{array}{l}\text { Exploratoria/ } \\
\text { Analítica }\end{array}$ & Música signada \\
\hline
\end{tabular}

Plantean la música signada como una nueva forma de arte y reflexionan sobre la pertinencia de utilizar el término música signada, en lugar de otras denominaciones sobre las experiencias relativas a este ámbito.

\begin{tabular}{|l|l|l|l|l|}
\hline Cripps, J. H, & Inglés & -Artículo de revista & Descriptiva/Ana- & Música signada \\
Rosenblum, & & lítica & \\
$\begin{array}{l}\text { E. Small, A. } \\
\text { \& Supalla, } \\
\text { S. (2017) }\end{array}$ & & -Google Académico y & & \\
\hline
\end{tabular}

Los participantes del "Signed Music Project" investigan formas de lo que llaman música signada y exploran los elementos musicales básicos en la modalidad signada. Definen este tipo de música como "una actuación viso-gestual que muestra elementos musicales", y es totalmente independiente de la experiencia auditiva. Su análisis preliminar muestra que la música signada incluye tres de los cinco elementos musicales básicos: el ritmo, la textura y el timbre. Falta la melodía y la armonía.

\begin{tabular}{|l|l|l|l|l|}
\hline $\begin{array}{l}\text { Peñalba, A., } \\
\text { Moriyón, C. } \\
\text { Luque, S. }\end{array}$ & Español \\
Cabezas, G. & -Actas de Congreso & $\begin{array}{l}\text { Descriptiva/Ana- } \\
\text { (2017) }\end{array}$ & -Manual & $\begin{array}{l}\text { Música y LS Con- } \\
\text { ceptualización de la } \\
\text { música a través de la } \\
\text { metáfora. Recursos }\end{array}$ \\
& & & & $\begin{array}{l}\text { lingüísticos emplea- } \\
\text { dos por oyente para } \\
\text { hacer llegar la músi- } \\
\text { ca al sordo. }\end{array}$ \\
\hline
\end{tabular}

Explica cómo las personas sordas se distancian de la música por su dificultad para percibirla, más que por su dificultad para aprehenderla. Las metáforas sirven de recurso pedagógico para acercar la música a las personas sordas a través de mapeos cognitivos que les ayuden a vincular lo abstracto del fenómeno musical con la experiencia corporal más concreta mediante el empleo de su propia lengua de signos. Es imprescindible tener competencias en música y conocer las obras instrumentales que se interpretan en LS para que resulte una interpretación de calidad.

\begin{tabular}{|l|l|l|l|l|}
\hline $\begin{array}{l}\text { Serna y } \\
\text { Piñera } \\
(\mathbf{2 0 1 8 )}\end{array}$ & Español & $\begin{array}{l}\text {-Acta de congreso } \\
\text {-Google Académico }\end{array}$ & $\begin{array}{l}\text { Descriptiva/Ana- } \\
\text { lítica }\end{array}$ & Canciones en LSE \\
\hline
\end{tabular}


Música más lengua de signos: una revisión de la literatura

Nadal-García, Icíar · López-Casanova, M. Belén •Juan-Morera, Borja

Explican cómo la interpretación de canciones en lengua de signos española (LSE) es un símbolo de comunicación que transmite sentimientos y significados extraídos de las canciones en lenguas orales. Defienden que la interpretación de canciones en LSE es el único medio por el que la comunidad sorda puede acceder al mundo de la música y describen el proceso que se debe llevar a cabo para realizar estas interpretaciones.

\begin{tabular}{|l|l|l|l|l|}
\hline $\begin{array}{l}\text { Peñalba, A. } \\
\text { Morillón, } \\
\text { C.,y Luque, } \\
\text { S. (2018) }\end{array}$ & Español & -Artículo de revista & $\begin{array}{l}\text { Descriptiva/Ana- } \\
\text { lítica Revisión de } \\
\text { proyectos }\end{array}$ & $\begin{array}{l}\text { Música instrumental } \\
\text { en LS }\end{array}$ \\
\hline
\end{tabular}

Justifican la música como un fenómeno multimodal donde las metáforas empleadas para conceptualizar fenómenos musicales son producto de metaforizaciones de la propia LS y las experiencias corporales se regulan a través de las propiocepciones que intervienen en la percepción y la autoconsciencia.

\begin{tabular}{|l|l|l|l|l|}
\hline $\begin{array}{l}\text { Nadal, I., } \\
\text { López, M.B., } \\
\text { Juan, B. y } \\
\text { Fernández, } \\
\text { C. (2018b) }\end{array}$ & Español & -Capítulo de libro & $\begin{array}{l}\text { Descriptivo } \\
\text { Experiencia/ } \\
\text { Proyecto }\end{array}$ & Música coral en LS \\
\hline
\end{tabular}

A través del coro inclusivo (constituido por personas con y sin diversidad funcional, y que cantan e interpretan con LS) se promueve y garantiza la sensibilización de la sociedad sobre la importancia de la igualdad de oportunidades. Las actividades de este coro contribuyen a la atención a la diversidad y la cohesión e inclusión social de sus componentes. Además de incidir en la mejora de la adquisición de competencias musicales, emocionales y sociales.

\begin{tabular}{|l|l|l|l|l|}
\hline $\begin{array}{l}\text { Nadal, l., } \\
\text { López, M.B., } \\
\text { Juan, B. y } \\
\begin{array}{l}\text { Fernández, } \\
\text { C. (2018a) }\end{array}\end{array}$ & Español & -Capítulo de libro & $\begin{array}{l}\text { Cualitativo Es- } \\
\text { tudio }\end{array}$ & Música coral en LS \\
\hline
\end{tabular}

La experiencia de cantar en un coro inclusivo, en el que participan personas con y sin diversidad funcional, y que cantan e interpretan en LS, crea unos vínculos tanto afectivos como sociales que traspasan el espacio del coro y que, al mismo tiempo, contribuyen al conocimiento de uno mismo y de los demás, a una mejora de las habilidades sociales y al desarrollo de valores fundamentales como pueden ser el respeto a la diferencia o la tolerancia. Igualmente, al utilizar la voz de forma colectiva, se genera un sentimiento profundo de igualdad y equidad entre los miembros del coro.

\begin{tabular}{|l|l|l|l|l|}
\hline $\begin{array}{l}\text { Beijer, N. C. } \\
\text { (2018) }\end{array}$ & Inglés & - Tesis de Máster & Investigación et- & $\begin{array}{l}\text { Canciones en LS. } \\
\text { nográfica explo- } \\
\text { Música en la cultura } \\
\text { Sorda holandesa. } \\
\end{array}$ \\
& & -Google Académico & $\begin{array}{l}\text { Entrevistas } \\
\text { semiestructura- } \\
\text { das y encuestas } \\
\text { online. }\end{array}$ & \\
\hline
\end{tabular}

Los hallazgos de esta investigación contribuyen a la comprensión de los aspectos de una práctica musical para sordos y, al mismo tiempo, invitan a explorar más a fondo las posibilidades que surgen al combinar estudios de música, estudios de discapacidad y estudios culturales para Sordos. El "baile de signos" es la práctica de traducir la letra de las canciones a la LS. 
Música más lengua de signos: una revisión de la literatura

Nadal-García, Icíar · López-Casanova, M. Belén ·Juan-Morera, Borja

\begin{tabular}{|l|l|l|l|l|}
\hline $\begin{array}{l}\text { Best, K. E. } \\
\text { (2018) }\end{array}$ & Inglés & $\begin{array}{l}\text {-Artículo de revista esp. } \\
\text { cultura Sorda } \\
\text {-Manual }\end{array}$ & $\begin{array}{l}\text { Investigación } \\
\text { etnográfica }\end{array}$ & $\begin{array}{l}\text { Canciones en LS. Ar- } \\
\text { tistas sordos a través } \\
\text { del Hip Hop }\end{array}$ \\
\hline
\end{tabular}

Analiza cómo los artistas sordos de Hip-hop adaptan el estilo de la música convencional para compartir de otro modo normas musicales centradas en la audición y así ampliar experiencias musicales a otros reinos sensoriales del cuerpo. De este modo, se abre la puerta a nuevas prácticas musicales para sordos y oyentes, lo que no solo allana el camino para futuros músicos sordos, sino que también ilustra la vibrante experiencia de la música en la cultura Sorda.

\begin{tabular}{|l|l|l|l|l|}
\hline $\begin{array}{l}\text { Dunn, } \mathbf{R .} \\
\text { (2018) }\end{array}$ & Inglés & $\begin{array}{l}\text {-Artículo de revista esp. } \\
\text { cultura Sorda } \\
\text {-Manual }\end{array}$ & $\begin{array}{l}\text { Descriptivo/Ana- } \\
\text { lítico }\end{array}$ & Canciones en LS \\
\hline
\end{tabular}

Reflexiona sobre el papel de la música en la comunidad Sorda y la forma en la que estas personas podrían intervenir en los espacios religiosos que emplean el canto como forma de oración. Propone un nuevo estilo musical, el Deaf beats, y acerca la música a la comunidad Sorda a través de la LS, para la cual demanda una mayor investigación.

\begin{tabular}{|l|l|l|l|l|}
\hline $\begin{array}{l}\text { Pirone, J. } \\
\text { (2018) }\end{array}$ & Inglés & $\begin{array}{l}\text {-Artículo de revista esp. } \\
\text { cultura Sorda } \\
\text {-Manual }\end{array}$ & $\begin{array}{l}\text { Descriptivo/Ana- } \\
\text { lítico }\end{array}$ & Música signada \\
\hline
\end{tabular}

Ofrece una discusión reflexiva sobre el lugar de la música en el aprendizaje de la LS. Estudia sus implicaciones pedagógicas en la traducción de canciones a estudiantes de LS preocupados por la apropiación cultural de esta lengua; Trata sobre las limitaciones que confrontan el campo de la enseñanza de LS con la música, ya que considera que pocos docentes tienen ambas competencias.

\begin{tabular}{|c|c|c|c|c|}
\hline $\begin{array}{l}\text { Listman, J. } \\
\text { Loeffler, S. } \\
\text { C. \& Timm, } \\
\text { R. L. (2018) }\end{array}$ & Inglés & $\begin{array}{l}\text {-Artículo de revista } \\
\text { especializada en cultura } \\
\text { Sorda } \\
\text {-Manual }\end{array}$ & $\begin{array}{l}\text { Descriptivo/Ana- } \\
\text { lítico }\end{array}$ & Canciones en LS \\
\hline
\end{tabular}

Destacan las tensiones que emergen en la comunidad Sorda sobre el lugar que debe ocupar la música en espacios culturales destinados a este colectivo, así como la idea de que la traducción de canciones y las complejidades de traducir música trasciende el lenguaje.

\begin{tabular}{|l|l|l|l|l|}
\hline $\begin{array}{l}\text { Buchholz, } \\
\text { N. (2018) }\end{array}$ & Inglés & $\begin{array}{l}\text {-Artículo de revista } \\
\text { especializada en cultura } \\
\text { Sorda } \\
\text {-Manual }\end{array}$ & $\begin{array}{l}\text { Descriptivo/Ana- } \\
\text { lítico }\end{array}$ & $\begin{array}{l}\text { Música signada. Mú- } \\
\text { sica y cultura Sorda }\end{array}$ \\
\hline
\end{tabular}

Ofrece una exploración acerca de su relación personal con la música como estudiante sordo. Él, como muchos otros, asumió que la música no era nativa de las personas sordas, descartando la idea de poder llegar a apreciarla y mucho menos de producir creaciones musicales para el consumo. Sin embargo, se acerca a la experiencia de ASL y canciones de percusión, reclamando un mayor nivel de investigación sobre este tema.

\begin{tabular}{|l|l|l|l|l|}
\hline $\begin{array}{l}\text { Robinson, } \\
\text { O. (2018) }\end{array}$ & Inglés & $\begin{array}{l}\text {-Artículo de revista } \\
\text { especializada en cultura } \\
\text { Sorda } \\
\text {-Google Académico }\end{array}$ & $\begin{array}{l}\text { Descriptivo/Ana- } \\
\text { lítico }\end{array}$ & $\begin{array}{l}\text { Música signada. Mú- } \\
\text { sica y cultura Sorda }\end{array}$ \\
\hline
\end{tabular}


Música más lengua de signos: una revisión de la literatura

Nadal-García, Icíar · López-Casanova, M. Belén ·Juan-Morera, Borja

Expone las ideas principales de un estudio realizado por nueve autores en el que se analiza como la música y las personas Sordas han transformado el concepto y la opinión que tenían sobre la cultura musical Sorda.

\begin{tabular}{|l|l|l|l|l|}
\hline Silvestri, J., & Inglés & -Artículo de revista & Descriptivo/Ana- & $\begin{array}{l}\text { Música signada. } \\
\text { Ehrenberg, } \\
\text { H., Dick, L. }\end{array}$ \\
$\begin{array}{l}\text { \& Shim, P. } \\
\text { (2018) }\end{array}$ & & $\begin{array}{l}\text { especializada en cultura } \\
\text { Sorda }\end{array}$ & lítico & \\
personas sordas
\end{tabular}

Defienden que la música debe de ser un fenómeno multimodal y multilingüe que proporcione múltiples medios de representación y expresión, teniendo en cuenta el pensamiento de la cultura Sorda. Elementos como la LS, los movimientos rítmicos o las nuevas tecnologías abren caminos más inclusivos a la educación musical.

\begin{tabular}{|l|l|l|l|l|}
\hline $\begin{array}{l}\text { Cripps, J. H. } \\
\text { (2018) }\end{array}$ & Inglés & $\begin{array}{l}\text {-Artículo de revista } \\
\text { especializada en cultura } \\
\text { Sorda } \\
\text {-Google Académico }\end{array}$ & $\begin{array}{l}\text { Descriptivo/Ana- } \\
\text { lítico } \\
\text { Estudio etnográ- } \\
\text { fico }\end{array}$ & Música signada \\
\hline
\end{tabular}

Opina que aquellas personas, sordas u oyentes, que decidan signar música deben tener en cuenta la cultura Sorda y asegurarse de que su investigación musical es auténtica, pues de lo contrario podría considerarse apropiación cultural.

\begin{tabular}{|l|l|l|l|l}
\hline $\begin{array}{l}\text { Begue, J. } \\
\text { \& Cripps, } \\
\text { Janis }\end{array}$ & Inglés & $\begin{array}{l}\text {-Artículo de revista } \\
\text { especializada en cultura } \\
\text { Sorda }\end{array}$ & $\begin{array}{l}\text { Descriptivo/Ana- } \\
\text { lítico }\end{array}$ & Música signada \\
(2018) & & -Google Académico & & \\
\hline
\end{tabular}

Distinguen entre traducción de canciones y música signada y describe, desde su experiencia personal con la comunidad Sorda y oyente, el proceso a seguir para la realización de un videoclip con música en LS.

\begin{tabular}{|l|l|l|l|l|}
\hline $\begin{array}{l}\text { Maulida, R. } \\
\text { P., Nayli- } \\
\text { Ihusna, Q. } \\
\text { \& Antula, P. } \\
\text { W. (2019) }\end{array}$ & & $\begin{array}{l}\text {-Artículo de revista } \\
\text { científica }\end{array}$ & $\begin{array}{l}\text { Descriptivo de } \\
\text { corte cualitativo }\end{array}$ & $\begin{array}{l}\text { Música signada } \\
\text { Experiencias comuni- } \\
\text { dad sorda y oyente }\end{array}$ \\
\hline
\end{tabular}

Muestran los pasos a seguir y las dificultades que pueden encontrarse a la hora de interpretar en LS una canción. El intérprete ha de ser consciente de que se trata de un proceso meticuloso que entraña diferentes desafíos, por lo que requiere de una gran motivación. Importancia del trabajo colaborativo de personas sordas y oyentes a la hora de buscar interpretaciones de la música en LS.

\begin{tabular}{|l|l|l|l|l|}
\hline $\begin{array}{l}\text { Juan, B., } \\
\text { Nadal, I.,y } \\
\text { López, M.B. } \\
\text { (2020) }\end{array}$ & Español & $\begin{array}{l}\text {-Artículo de revista } \\
\text { científica } \\
\text {-Scopus, WoS y Google } \\
\text { Académico }\end{array}$ & Cualitativo & $\begin{array}{l}\text { Música coral en LS. } \\
\text { Signado a 4 voces }\end{array}$ \\
\hline
\end{tabular}


Música más lengua de signos: una revisión de la literatura

Nadal-García, Icíar · López-Casanova, M. Belén ·Juan-Morera, Borja

Constatan como el signado de canciones a varias voces dota de significado al discurso y a la interpretación musical. Además, contribuye en aspectos técnicos del canto (la correcta emisión, la respiración, la proyección y/o el fraseo), puesto que la musicalidad del gesto acompaña la intención melódico-rítmica de la música, así como el carácter y/o los distintos matices.

Concluyen que el signado de obras polifónicas interpretadas por un coro, acerca la música a personas con y sin déficit auditivo puesto que la LS permite reforzar el concepto de polifonía de forma visual, pudiéndose apreciar el juego dialéctico entre las voces. Así mismo, la interpretación musical de la polifonía en LS favorece la inclusión y amplía el acceso a la cultura de las personas sordas. Reclaman más investigación sobre el tema.

\begin{tabular}{|c|c|c|c|c|}
\hline $\begin{array}{l}\text { Cripps, J. H., } \\
\text { Small, A., } \\
\text { Rosenblum, } \\
\text { E., Supalla, } \\
\text { S. J., Whyte, } \\
\text { A. K., \& Cri- } \\
\text { pps, J. S. (En } \\
\text { prensa) }\end{array}$ & Inglés & $\begin{array}{l}\text {-Capítulo de libro } \\
\text {-Manual }\end{array}$ & $\begin{array}{l}\text { Descriptiva/Ana- } \\
\text { lítica }\end{array}$ & Música signada \\
\hline
\end{tabular}

Reivindican la música signada como una nueva forma de arte que nace en manos de la comunidad Sorda, que incorpora su experiencia, sirviendo de inculturación natural y brinda apoyo a este colectivo. Opinan que la música signada es tan estética y agradable a los ojos como la música auditiva es agradable a los oídos.

\section{Resultados de los documentos redactados en español}

De los 11 artículos que se preseleccionaron en la primera búsqueda y una vez leído el documento completo, solo 7 fueron seleccionados. El primer artículo publicado en español sobre la temática estudiada, es una tesis de maestría de Andrade del año 2016. El resto de los documentos se localizan en revistas españolas, capítulos de libros y actas de congresos. Al realizar la revisión se percibió que, aunque en España sí que se realizaban prácticas que acercaban la música y la LSE (PEÑALBA et al., 2017; PEÑALBA et al., 2018; JUAN et al., 2020), no se encuentran documentos académicos que analicen dichas experiencias hasta 2017.

De estos 7 documentos solo uno de ellos es un artículo científico y se encuentra indexado en Scopus y WoS (JUAN et al., 2020). La metodología que emplea es cualitativa y utiliza diferentes instrumentos para la recogida de datos. Entre sus aportaciones se destaca que el signado de obras polifónicas interpretadas en LS 
acerca la música a personas con y sin déficit auditivo ya que la LS permite reforzar el concepto de polifonía de forma visual y apreciar el juego dialéctico entre las voces.

En cuanto a los métodos de investigación en los que se centran los documentos: dos de ellos utilizan enfoques cualitativos (JUAN et al., 2020; NADAL et al., 2018a); el resto utiliza metodologías descriptivas y analíticas (PEÑALBA et al., 2018; SERNA Y PIÑERA, 2017; ANDRADE 2016; PEÑALBA et al., 2017); y ninguno emplea métodos mixtos o cuantitativos.

Los temas de estudio se basan: tres en el coro y LS (2020, [2] 2018); uno sobre música en LS colombiana (2016); uno sobre canciones en LS (2017); uno sobre música en LSE; y uno sobre la música instrumental en LS (2018).

En referencia al lugar de publicación: dos aparecen en revistas especializadas (revista electrónica LEEME y Tabanque); dos en capítulos de libros (Editoriales Procompal y Octaedro); dos en actas de congresos (Actas de Congresos CNLSE de la LSE); y una tesis de maestría en Bogotá.

\section{Resultados de los documentos redactados en inglés}

De los 195 artículos seleccionados en la primera búsqueda únicamente 23 superaron los criterios de inclusión establecidos. El primer artículo relacionado con el objeto de estudio está fechado en 1985 y realiza una investigación de corte cualitativo en la que utiliza las entrevistas como herramienta de investigación. Concibe la interpretación de música en LS como una nueva profesión y acerca la cultura Sorda a los oyentes. Se trata de una revista científica indexada, pero el artículo no figura en ninguna de las bases de datos consultadas en la investigación, debido al año de publicación.

De los 23 documentos seleccionados 4 son capítulos de libros: 3 se encuentran en volúmenes especializados en sordos (BAHAN, 2006; CRIPPS et al., 2016; CRIPPS et al., en prensa) y 1 en un 
volumen sobre discapacidad (MALER, 2015). Localizamos una Tesis de Fin de Máster realizada en 2018, en la universidad de Ulrecht. La serie Deaf Arts Handbook (2015) dedica un volumen a la música. Los 17 restantes documentos son artículos de revistas. Nueve los encontramos en un número que la revista Journal of American Sign Languages \& Literatures dedicó a la música signada en 2018. Dos documentos fechados en 2017 en una revista especializada en ASL, sin indexación. Seis en revistas indexadas: 3 en revistas de musicoterapia (1999, 2004 y 2013), 1 en una revista especializada en LS (1985) y 2 en revistas con diferentes temáticas (2017 y 2019). Es de especial relevancia señalar algunas de las aportaciones extraídas de la consulta de estas investigaciones como es la constatación de que el signado y el ritmo visual aumenta la percepción musical (DARROW \& LOOMIS, 1999; MALER, 2013). Además, (CRIPPS et al., 2017) señalan que las actuaciones viso-gestuales muestran los elementos musicales y es independiente de las experiencias auditivas. Finalmente, Maulida et al., (2019) indican la importancia del trabajo colaborativo de personas sordas y oyentes a la hora de buscar interpretaciones de la música en LS.

La temática se centra en estudios sobre música signada (18 documentos) y canciones en LS (5 documentos). La mayoría (19) se publican en EE. UU., 6 en España, 1 en Colombia, 2 en Holanda, 1 en Noruega, 1 en Canadá.

Los métodos de investigación son principalmente cualitativos, descriptivos y analíticos. Se desarrollan mediante diferentes técnicas y procedimientos: relato etnográfico, análisis de videos, entrevistas semiestructuradas y observación directa. Ninguno de los estudios utiliza una metodología cuantitativa o mixta.

\section{Discusión y conclusiones}

A través de esta revisión documental se han analizado las contribuciones llevadas a cabo por diferentes autores en el ámbito de la música y la LS. La mayoría de los estudios son de corte cualitativo, 
descriptivo y analítico y ninguno de enfoque cuantitativo. Este análisis nos lleva a destacar las aportaciones que evidencian la contribución de la LS a la música como una experiencia artística, tanto para la comunidad Sorda como para la oyente.

Se constata la diferencia entre los términos música signada y música interpretada-traducida a la LS (BEGUE \& CRIPPS, 2018; CRIPPS \& LYONBLUM, 2017; CRIPPSET al., 2016; CANADIAN CULTURAL SOCIETY OF THE DEAF, 2015). La música signada es la realizada por los nativos Sordos. Para Cripps et al. (2016), cuando el signado del lenguaje y los componentes viso-gestual y multisensoriales se utilizan para expresar actuaciones artísticas dentro de la comunidad Sorda, el resultado se denomina Signed Music (música signada). Sin embargo, la música interpretada-traducida a la LS, es la que realizan las personas oyentes o la llevada a cabo por personas sordas y oyentes (mixta).

Según MALER (2015), la traducción de canciones a la LS es un fenómeno distinto a la música signada, ya que las personas oyentes signan las canciones de modo diferente que las sordas, utilizando distinta forma de comunicación. Las personas sordas tienden a usar múltiples técnicas de comunicación en sus presentaciones, mientras que las personas oyentes no lo hacen. Las canciones traducidas a la LS transcriben palabra a palabra (BEGUE \& CRIPPS, 2018). En este sentido Maulida et al. (2019), sugieren que para los nativos Sordos la LS es su herramienta de comunicación, por lo tanto, signan las canciones para crear "nueva música" en LS. Sin embargo, los oyentes que realizan estas prácticas se enfrentan a otros desafíos importantes. Bahan (2006), caracteriza a las canciones signadas como un subproducto de canciones en las que las personas sordas interaccionan con el mundo de la audición. No obstante, la música interpretada-traducida a la LS hace que esta experiencia tenga una perspectiva diferente, puesto que no solo consiste en la traducción de las canciones palabra por palabra, sino en la representación de los diferentes parámetros de la música a través de la expresión corporal. 
En el desarrollo de la música interpretada-traducida a la LS es importante que colaboren de forma activa y conjunta todos los agentes participantes (intérpretes, personas sordas y oyentes). Se espera que una fuerte conciencia y comprensión entre estas tres partes aumente la igualdad de oportunidades y la inclusión (MAULIDA et al., 2019). Es importante cerrar las brechas entre las personas sordas y los intérpretes de canciones en LS; deberían trabajar juntos para encontrar una interpretación adecuada y conocer sus intereses y necesidades (MICHEAL, 2011).

La música en LS es considerada como un nuevo arte multimodal inclusivo que hace que las experiencias perceptivas sean más significativas y que se perciban por el espectador más intensamente de una forma multisensorial (auditiva, visual y propioceptiva). Al mismo tiempo, mejora la percepción musical de los sordos y los oyentes, pues permite experimentar la música de nuevas formas (MALER, 2014). Favorece el proceso de implicación e inclusión de la comunidad Sorda y oyente, también conocido como proceso de inculturación. De acuerdo con Thompson, Graham, \& Russo (2005) y Platz \& Kopiez (2012), los aspectos visuales de la música influyen significativamente en la experiencia musical y, sin embargo, la música es a menudo concebida como una experiencia puramente auditiva por las personas oyentes.

Las redes sociales han favorecido la difusión y popularidad que están adquiriendo estas prácticas. El número de videos de música en LS ha ido aumentando de forma exponencial. Se han creado vídeos de canciones signadas en colaboración con músicos conocidos que alcanzan millones de visitas en las redes, y aumentan el reconocimiento y la valoración de la LS (MAULIDA et al., 2019). Con la difusión de estos videos de conciertos subidos a YouTube, Vimeo y otras plataformas digitales, se atrae a una audiencia internacional, se llega a personas sordas y oyentes, y contribuyen a desarrollar una cultura de inclusión después de que las actuaciones hayan concluido.

Existen encontradas opiniones acerca de si los sordos son capaces de disfrutar de la música (BEGUE \& CRIPPS, 2018; BUCHHOLZ, 
2018). Son desacuerdos de ida y vuelta dentro de la comunidad Sorda. La música en LS a través de su representación, no solo auditiva, puede llegar a multiplicar las emociones de las personas sordas y oyentes que se acerquen a este arte.

Tanto la comunidad Sorda como las personas oyentes que realizan estas prácticas, reconocen que tan apenas existe investigación y consideran importante fomentar la realización de nuevos estudios. Se necesita avanzar en las investigaciones para explorar y evidenciar los presuntos beneficios que aporta este nuevo arte de la música más la LS.

\section{Referencias}

ANDRADE, Fernando. Manos, miradas y silencios otros... Resignificaciones culturales hacia una música propiamente Sorda. (Tesis de Maestría inédita). Universidad Nacional de Colombia, 2016.176 p. BAHAN, Ben. Face-to-face tradition in the American Deaf Community. En: BAUMAN, H-Dirksen; NELSON, Jennifer L. y ROS, Heidi H. (Ed.). Signing the body poetic. California: University of California Press, 2006, p.21-50.

BEGUE, Jason \& CRIPPS, Janis. The Artwork of Video Editing in Signed Music. Journal of American Sign Languages \& Literatures, 2018, Disponible en <http://journalofasl.com/wp-content/uploads/ sites/8/2018/08/the-artwork-of-video-editing-in-signed-music.pdf>. Consultado el 18 de marzo de 2020.

BEIJER, Naomi. Unheard of: an Exploratory Ethnographic Research into the musical Practice of Deaf and hard of Hearing People in the Netherlands. (Tesis MA inédita). Utrecht University Netherlands, $2018.159 \mathrm{p}$.

BEST, Katelyn E. Musical Belonging in a Hearing-Centric Society: Adapting and Contesting Dominant Cultural Norms through Deaf Hip Hop. Journal of American Sign Languages \& Literatures, 
2018. Disponible en <http://journalofasl.com/wp-content/uploads/ sites/8/2018/06/best.pdf>. Acceso al 4 de abril de 2020.

BUCHHOLZ, Noah. Seeing Music? An Inquiry into the Place of Music in Deaf Culture. Journal of American Sign Languages \& Literatures, 2018. Disponible en <http://journalofasl.com/wp-content/uploads/ sites/8/2018/06/buchholz.pdf>. Consultado el 22 de mayo de 2020. CANADIAN CULTURAL SOCIETY OF THE DEAF (Series author). Signed music: Rhythm of the heart. Deaf Arts Handbook Series. Toronto, Ontario: Canadian Cultural Society of the Deaf, vol.2, 2015. Disponible en <https://slicanada.ca/wp-content/uploads/2017/09/642_ DeafArtsHandbook_Volume2_FINAL2015-1.pdf >. Consultado el 23 de abril de 2020

CRIPPS, Jody; ROSENBLUM, Ely \& SMALL, Anita. Music, Signed. En GERTZ, Genie; BOUDREAULT, Patrick (Eds.). The SAGE Deaf Studies Encyclopedia. Los Ángeles: SAGE Publications, 2016, p.703-706.

CRIPPS, Jody; LYONBLUM, Ely. Understanding the use of signed language for making music. Society For American

Sign Language Journal SASLJ, vol. 1. n.1, p.78-95, 2017.

Disponible en: <http://tigerprints.clemson.edu/cgi/viewcontent. cgi?article=1000\&context=saslj>. Consultado el 11 de mayo de 2020 . CRIPPS, Jody H. Ethnomusicology \& Signed Music: A Breakthrough. Journal of American Sign Languages \& Literatures, 2018. Disponible en <http://journalofasl.com/wp-content/uploads/sites/8/2018/08/ ethnomusicology_cripps.pdf >. Consultado el 3 de abril de 2020. CRIPPS, Jody H.; SMALL, Anita; ROSENBLUM, Ely; SUPALLA, Samuel J.; WHYTE, Aimee \& CRIPPS, Joanne S. Signed music and the deaf community. En CRUZ, A (Ed.). Culture, deafness \& music: Disability studies and a path to social justice. Rotterdan, N L: Sense Publishers. (In press). Disponible en https://www.academia.edu/33661954/SIGNED_ MUSIC_AND_THE_DEAF_COMMUNITY_Book_Chapter_. Consultado el 23 de mayo de 2020.

CURTIS, Sandra; MERCADO, Chesley. Community Music Therapy for Citizens with Developmental Disabilities. Voices: A World Forum 
For Music Therapy, vol.4, n.3 2004. Disponible en <https://doi. org/10.15845/voices.v4i3.185>. Consultado el 5 de mayo de 2020. CURTIS, Sandra. Celebrating the Creative Spark: Community Building through Music Therapy. En BRIAN MCBAY (Ed.). Proceedings of the Canadian Association for Music Therapy 30th Annual Conference. Waterloo, ON: Canadian Association for Music Therapy, 2004, p. 17-21. DARROW, Alice-Ann; LOOMIS, Diane Merchant. Music and Deaf Culture: Images from the Media and Their Interpretation by Deaf and Hearing Students. Journal of Music Therapy, Oxford, vol. 36, n.2, p. 88109.1999

DUNN, Renca. Deaf People and the Role of Music in the Churches. Journal of American Sign Languages \& Literatures, 2018. Disponible en <http://journalofasl.com/wpcontent/uploads/sites/8/2018/08/ rencadunn.pdf $>$. Consultado el 28 de abril de 2020.

EGBERT, Lisalee A. Is Silence Music to the Eye? A Review of Signed Music: A Symphonious Odyssey. Society for American Sign Language Journal SASLJ, vol.1, 2017. Disponible en <http://tigerprints.clemson. edu/cgi/viewcontent.cgi?article=1000\&context=saslj>. Consultado el 2 de marzo de 2020.

FEDERACIÓN MUNDIAL DE PERSONAS SORDAS (WFD). Lengua de signos y derechos para todos. En: XVIII CONGRESO MUNDIAL DE PERSONAS SORDAS, 2019. Disponible en <https://bit.ly/2ZoGH6W>. Consultado el 21 de junio de 2020.

HOLMES, Jessica A. Expert Listening beyond the Limits of Hearing: Music and Deafness. Journal of The American Musicological Society, n.70, 2017. Disponible en <https://doi.org/10.1525/jams.2017.70.1.171>. Consultado el 19 de abril de 2020.

JUAN, Borja; NADAL, Icíar y LÓPEZ, M. Belén. Música y lengua de signos a cuatro voces, una experiencia educativa y musical para la inclusión. Revista Electrónica de Leeme, n.45, 2020. Disponible en <https://ojs. uv.es/index.php/LEEME/index>. Consultado el 6 de marzo de 2020. KIM, Christine Sun. The Enchanting Music of Sign Language. En: TEDTALK, 2015. Disponible en <https://www.ted.com/talks/ 
christine_sun_kim_the_enchanting_music_of_sign_language/ transcript?language=en>. Consultado el 28 de mayo de 2020.

LISTMAN, Jason; LOEFFLER, Summer Crider \& TIMM, Rosa Lee. Deaf Musicality and Unearthing the Translation Process. Journal of American Sign Languages \& Literatures, 2018. Disponible en $<$ http://journnnalofasl.com/wp-content/uploads/sites/8/2018/08/deafmusicality.pdf>. Consultado el 2 de junio de 2020.

MALER, Anabel. Songs for Hands: Analyzing Interactions of Sign Language and Music. Journal of The Society For Music Theory, vol.19, n.1 2013. Disponible en <http://mtosmt.org/issues/mto.13.19.1/ mto.13.19.1.maler.html>. Consultado el 9 de mayo de 2020.

MALER, Anabel. Musical Expression among Deaf and Hearing Song Signers. En HOWE, Blake; JENSEN-MOULTON, Stephanie; LERNER, Neil; Straus, Joseph (Eds.). The Oxford Handbook of Music and Disability Studies, 2015, p. 73-91. Disponible en <https://doi.org/10.1093/ oxfordhb/9780199331444.013.4>. Consultado el 9 de marzo de 2020. MAULIDA, Rulli Putri; NAYLILHUSNA, Qonita; ANTULA, Pungky W. Promoting Sign Language by Digitizing Song Interpretation using Indonesian Sign Language in Social Media. Advances in Social Science, Education and Humanities Research, vol. 254, p. 331-337, 2019. Disponible en <https://doi.org/10.2991/conaplin-18.2019.278>. Consultado el 4 de mayo del 2020.

NADAL, Icíar; LÓPEZ, M. Belén; FERNÁNDEZ, Carmen; JUAN, Borja. El coro Cantatutti: una práctica musical inclusiva en la Universidad de Zaragoza. En: ROIG-VILA, Rosabel (Ed.). El compromiso académico y social a través de la investigación e innovación educativas en la Enseñanza Superior. Barcelona: Octaedro, 2018. p.859-869.

NADAL, Icíar; LÓPEZ, M. Belén; FERNÁNDEZ, Carmen; JUAN, Borja. Cantar para incluir. En: LAG, Nieves (coord.), Educación musical Recursos para el cambio Metodológico. Almería: Procompal, 2018b. p.165-172.

PEÑALBA, Alicia; MORIYÓN, Carlos; LUQUE, Sonia; CABEZAS, Gonzalo. La metáfora conceptual como recurso pedagógico musical para personas sordas y con discapacidad auditiva. En: ACTAS DEL CONGRESO CNLSE 
DE LA LENGUA DE SIGNOS ESPAÑOLA. Madrid: Real Patronato sobre Discapacidad, 2017, p. 87-108.

PEÑALBA Alicia, MORIYÓN, Carlos; LUQUE, Sonia. Más que sonido: interpretación de música instrumental en lengua de signos para las personas sordas. Tabanque. Revista Pedagógica, vol. 31, p. 94-107, 2018. Disponible en <https://doi.org/10.24197/trp.31.2018.94-107>. Consultado el 7 de abril de 2020.

PIRONE, John. Does Music Have a Place in ASL Pedagogy? Journal of American Sign Languages \& Literatures, 2018. Disponible en <http:// journalofasl.com/wpcontent/uploads/sites/8/2018/06/pirone.pdf>. Consultado el 9 de mayo de 2020.

PLATZ, Friedrich \& KOPIEZ, Reinhard. When the Eye Listens: A Metaanalysis of How Audio-visual Presentation Enhances the Appreciation of Music Performance. Music Perception: an Interdisciplinary Journal, Oakland, vol. 30, p. 71-83, 2012. Disponible en <https://doi.org/ doi:10.1525/mp.2012.30.1.71>. Consultado el 7 de mayo de 2020. ROBINSON, Octavian. Deafening Music: Transcending Sound in Musicking. Journal of American Sign Languages \& Literatures, 2018. Disponible en <http://journalofasl.com/wpcontent/uploads/ sites/8/2018/06/pirone.pdf>. Consultado el 11 de abril de 2020. SERNA, Eva María; PIÑERA, Pedro. La interpretación de canciones en lengua de signos española: problemas traductológicos. En ESTEBAN, Maria Luz (Coord.), ACTAS DEL CONGRESO CNLSE DE LA LENGUA DE SIGNOS ESPAÑOLA. Madrid: Real Patronato sobre Discapacidad, 2018, p.65-75.

SERRAT MANÉN, Jordi; FERNÁNDEZ-VIADER, M. Pilar. Una aproximación a los referentes informativos de las personas sordas. Revista Española de Discapacidad, V. 1, p. 179-194, 2013. Disponible en <https://www. cedd.net/redis/index.php/redis/article/view/41/33>. Consultado el 14 de mayo de 2020.

SILVESTRI, Julia; EHRENBERG, Hannah; DICK, Lauren \& SHIM, Paul. Universal Design for Music: Exploring the Intersection of Deaf Education and Music Education. Journal of American Sign Languages \& 
Literatures, 2018. Disponible en <http://journalofasl.com/wp-content/ uploads/sites/8/2018/06/udl.pdf>. Consultado el 23 de mayo de 2020.

SUTHERLAND, Elaine. Music to Their Eyes: Song-to-Sign Interpreting at the Hudson Clearwater Festival. Sign Language Studies, vol. 49, p. 363-373, 1985. Disponible en < https://doi.org/10.1353/sls.1985.0012>. Consultado el 12 de abril de 2020.

TEJADA, Jesús. Música y mediación de la tecnología en sus procesos de aprendizaje. Educación XX1, Madrid, V. 7, p. 15-26, 2004. Disponible en $<$ https://doi.org/10.5944/educxx1.0.7.327>. Consultado el 17 de mayo de 2020.

THOMPSON, William Forde; GRAHAM, Phil; RUSSO, Frank A. Seeing music performance: Visual influences on perception and experience. Semiotica, 156, p. 203-227, 2005. Disponible en <https://doi. org/10.1515/semi.2005.2005.156.203>. Consultado el 27 de mayo de 2020.

\section{Agradecimentos}

Los autores quieren hacer constar como agradecimiento que este estudio ha sido posible gracias al convenio de colaboración entre el departamento de Ciudadanía y Derechos Sociales del Gobierno de Aragón y la Universidad de Zaragoza, en el marco de la Cátedra institucional "Música e Inclusión para el cambio social"; si bien para la realización de este estudio no se ha contado con financiación.

\section{Contribuiç̧ão de autoria}

Los tres autores han diseñado el método de estudio y las técnicas e instrumentos para su desarrollo y han colaborado en la revisión sistemática de los documentos. La discusión y conclusiones ha sido realizada por los tres investigadores, considerando positivo el enriquecimiento que ha supuesto la complementariedad 
de visiones. Todos los autores indican que este documento no ha sido publicado anteriormente ni ha sido enviado a otra revista. Asimismo, ha sido revisado para asegurar el anonimato en la evaluación por pares.

\section{Publisher}

Universidade Federal de Goiás. Escola de Música e Artes Cênicas. Programa de Pós-graduação em Música. Publicação no Portal de Periódicos UFG.

As ideias expressadas neste artigo são de responsabilidade de seus autores, não representando, necessariamente, a opinião dos editores ou da universidade. 